\title{
Subjective Outcome Measures of Drug Efficacy
}

\author{
Soizic Courcier-Duplantier, ${ }_{1}^{1}$ Bruno Falissard, ${ }^{2}$ Pierre Fender ${ }^{3}$ and the Participants in \\ Round Table No. 6, Giens XVIII ${ }^{* *}$
}

1 Roche Pharma, Neuilly-sur-Seine, France

2 INSERM U472, Hôpital Paul Brousse, Villejuif, France

3 CNAMTS, Paris, France

\begin{abstract}
The use of subjective outcome measures for assessing drug efficacy varies according to the disease in question. Subjective outcome measures used to complement an objective outcome measure can clearly claim the status of a main outcome measure. The validation of an instrument follows an appropriate methodology that focuses on two points: the methods used for its construction are set out and its performance is evaluated in a study. In drug evaluation, the importance of the subjective outcome measure should be discussed, depending on the aim and the disease. The methodology of the study obeys the same rules as when an objective outcome measure is used. The issue of the clinical significance of the results should be broached and discussed. Subjective outcome measures deserve to be considered in the evaluation of the drug because they provide a different and complementary perspective on the disease and the patient, both at the time of obtaining the marketing authorisation or when reassessing a drug.
\end{abstract}

Keywords: subjective, efficacy, instrument, validation, evaluation

Text received May 6, 2003; accepted May 26, 2003

A great many measures are implemented during a clinical trial and particular attention must be paid to the outcome measures. ${ }^{[1]}$ Various types of variables can be used, be they 'objective' or 'subjective'. The adjective 'subjective' can raise doubts about the presumed scientific rigour that is expected of an outcome measure for drug efficacy.

The aim of this round table was to review the use of subjective outcome measures in evaluating drug efficacy.

In the first place, it seems essential to reach a consensus regarding the definition of 'subjective outcome measure'. The definition adopted was as follows: any outcome measure for which the patient is considered as a thinking subject. This is the case for sadness, fatigue or breathlessness. Conversely, peak expiratory flow rate and blood pressure are objective outcome measures, because they are associated more with organs or physiological systems than with a patient who is considered as a thinking subject. The patient's perception is recorded using a questionnaire or a scale. ${ }^{[2]}$

Drug efficacy can be evaluated in all therapeutic fields using subjective outcome measures, and many instruments exist for this purpose.

Three objectives were selected by the members of the round table: (i) to discuss in which situations subjective outcome measures of drug efficacy are used; (ii) to describe the scales, their metrological properties and their validation methods; and (iii) to broach the subject of the importance of these outcome measures

$\dagger$ For a list of participants, please see the end of the article.

* Articles, analyses and proposals arising from the Giens Clinical Pharmacology Meetings are the responsibility of their authors and do not reflect the views held by their supervisory organisation. 
at the time of obtaining the marketing authorisation (MA) and when reassessing drugs after their release onto the market.

\section{Subjective Outcome Measures: In Which Situations Are They Used?}

An investigation of the various disease situations led to the conclusion that the use of subjective outcome measures for assessing efficacy varies, depending on the disease being studied. In reality, there is a kind of 'spectrum' starting from:

- situations where there are only objective outcome measures,

- followed by situations where the use of a mixture of objective and subjective outcome measures poses a number of problems that vary according to the disease in question,

- and, finally, situations where only subjective outcome measures exist.

We chose to deal with these different situations by means of examples, presenting a few representative diseases and setting out each outcome measure in relation to the others (comparing the objective response and the subjective response, weighting the objective response with the subjective response, and considering the pertinence of the effect size, etc.).

\subsection{Situations Where Only Objective Outcome Measures Exist: Vaccine Development}

In vaccine development, there are only objective outcome measures. If no link is established between antibody titre and efficacy (whooping cough, herpes), the outcome measure adopted will be the number of times the vaccine fails (number of clinical cases notified in the vaccinated population). When a clear link is established between the antibody titre and efficacy, efficacy is evaluated on the single outcome measure of immunogenicity (diphtheria, tetanus, polio). In the longer term, and notably during post-MA reassessment, the decrease in the incidence of the disease then becomes a good outcome measure of the vaccine's efficacy. These objective variables are either qualitative (presence or absence of the disease), or quantitative (antibody titre). Nevertheless, trials on vaccine efficacy are limited by their inability to predict the performance of the vaccine when it is introduced into public health practice, and do not provide certain types of information such as, for example, the level of uptake of the vaccination. ${ }^{[3]}$

\subsection{Situations Where Both Objective and \\ Subjective Outcome Measures Exist: Rheumatology, Chronic Obstructive Lung Disease and Oncology}

\subsubsection{Rheumatology}

In rheumatology, the following measures coexist:

- objective outcome measures: clinical measures (oedema, inflammatory signs, mobility), laboratory measures (erythrocyte sedimentation rate, C-reactive protein), and imaging measures (narrowed joint space);

- $\quad$ subjective outcome measures: symptoms (pain), collections of symptoms (index), global assessment, quality of life;

- mixed outcome measures that combine both objective and subjective outcome measures.

Thus, when evaluating the efficacy of a treatment for osteoarthritis, one can use an objective outcome measure (measurement of cartilage destruction) and a subjective outcome measure (evaluating pain by means of a self-assessment scale (visual analogue scale [VAS]). There are also multi-symptom scales and algofunctional indices. Rheumatologists from various countries evaluated the relative importance of these outcome measures at a meeting of the OMERACT (Outcome Measures in Rheumatology Clinical Trials [a scientific society that is part of the International League Against Rheumatism]). They ranked pain in the first place, with physical function next (algofunctional index), followed by the global assessment by the patient and, finally, imaging outcome measures. ${ }^{[4]}$

In rheumatoid arthritis, an index (the American College of Rheumatology [ACR] 20, 50, 70 ${ }^{[5]}$ ) combining objective outcome measures (joint stiffness) and subjective outcome measures (pain, global assessment by the doctor and by the patient) was recognised as the main outcome measure by the ACR and the European League Against Rheumatism, and is incorporated in the guidelines for development.

This raises many questions: Why is it necessary to use a subjective outcome measure when one has an objective outcome measure? Similarly, what is the value in having an objective outcome measure when one has a subjective outcome measure? How do these outcome measures fit together? What is the correlation between them? How does one assess the size of the effect? Thus, in osteoarthritis, what is the importance of pain or of using an algofunctional index compared with measuring cartilage destruction?

\subsubsection{Chronic Obstructive Lung Disease}

At present, there is no primary therapy for chronic obstructive lung disease (COLD) and the available treatments are mainly aimed at relieving symptoms. The European Agency for the Evaluation of Medicinal Products (EMEA) "Points to consider" ${ }^{[6]}$ rec- 
ommends first evaluating drug efficacy by means of an objective outcome measure, i.e. forced expiratory volume in one second $\left(\mathrm{FEV}_{1}\right)$ and, second, using a subjective outcome measure, the St George's Respiratory Questionnaire. These two variables must be regarded as the main outcome measures. There are many secondary outcome measures, either objective (lung function test, oxyhaemoglobin saturation, $\mathrm{CO}_{2}$ assay, exacerbation rate) or subjective (symptom scales, quality of life, treadmill test). In terms of preventing progression of the disease, the EMEA "Points to consider" ${ }^{[6]}$ recommends using an objective outcome measure, the $\mathrm{FEV}_{1}$, whereas the exacerbation rate over 6 months or 1 year accompanied by subjective outcome measures such as symptom scales, notably measuring dyspnoea or effort tolerance, would seem to be essential and relevant assessments in COLD. The $\mathrm{FEV}_{1}$ is somewhat insensitive to the pharmacological effect of drugs. It is weakly correlated with symptoms (dyspnoea), effort tolerance and quality of life. In addition, it decreases in a physiological manner, and more or less quickly, depending on whether the subject is a smoker. A primary therapy for COLD would therefore need to demonstrate an ability to slow the decline in $\mathrm{FEV}_{1} \cdot{ }^{[7]}$ These limitations provide a good explanation of why "Points to consider" $[6]$ recommends a second main outcome measure, this time subjective. In this situation, although the objective outcome measure has limits it must nevertheless be considered the primary main outcome measure and, although the subjective outcome measure seems relevant, it cannot be considered the sole main outcome measure.

Although the proposed objective outcome measure is not completely satisfactory, this example raises the issue of the recognition of subjective outcome measures in drug evaluation.

Why would the use of subjective outcome measures not be sufficient when evaluating treatments that are purely aimed at relieving symptoms?

\subsubsection{Oncology}

In oncology, when considering the registration of a drug, the expectations of the authorities are clearly defined. The recognised outcome measure is an objective outcome measure, whether it is the disease-free interval with no exacerbation and no recurrence, the disease-free interval until exacerbation or recurrence, or the rate of objective response (reduction of tumour size according to validated outcome measures [EMEA "Note for Guidance" $\left.{ }^{[8]}\right]$ ). However, it is preferable to add a measure of the service provided by a treatment to the simple notion of survival, and it is therefore indispensable to weight the efficacy of a therapy with its impact on quality of life. ${ }^{[9]}$ If patients are not cured, their expectations and those of the prescribing physicians are that the treatment should prolong life with adverse effects. In such cases, evaluating the treatment using subjective outcome measures (reduction of symptoms) therefore has an important place. From the point of view of the pharmaceutical industry, consideration of subjective outcome measures together with objective outcome measures encourages innovation.

Thus, for example, pancreatic cancer is often diagnosed at an advanced stage because the first symptoms are non-specific, and the rate of survival at 5 years is only $4 \%$, at whatever stage the cancer is diagnosed.

During the 1990s, Lilly developed a new cytotoxic agent for this indication called gemcitabine. In two key trials, its efficacy was measured by taking an intermediate outcome measure as the main outcome measure, namely 'clinical benefit response' (the clinical improvement measured in terms of analgesic intake, pain intensity, performance and changes in weight). These trials also measured survival, disease progression and tumour growth. In spite of doubts about its efficacy in terms of the objective outcome measures, the US FDA granted approval for its registration on the basis of the 'clinical benefit', notably taking into account the lack of alternative therapies for this indication.

Other examples in oncology show that the symptomatic response and the objective response are not always correlated. ${ }^{[9]}$

In this discipline, it seems necessary to weight survival results with subjective outcome measures that assess improvement in symptoms or quality of life.

Perhaps the use and recognition of subjective outcome measures in the evaluation of treatments in oncology could also make it possible to identify the target population who would benefit the most from the treatment and to define the best therapeutic strategies.

\subsection{Situations Where Only Subjective Outcome Measures Exist: Alzheimer's Disease}

In diseases of the central nervous system, subjective outcome measures are used more than objective outcome measures. This is true whether we are talking about psychiatric conditions (depression, anxiety, schizophrenia) or neuropsychiatric diseases (Parkinson's, Alzheimer's). ${ }^{[10]}$ Indeed, in these conditions it is necessary to incorporate the social dimension as well as the symptoms specific to the disease, and to have multidimensional methods of evaluation. ${ }^{[11]}$

In Alzheimer's disease, the diagnostic criteria combine the Mini-Mental State Examination (MMSE), the Diagnostic and Statistical Manual of Mental Disorders - Fourth Edition (DSMIV), and clinical criteria based exclusively on questioning the patient or the people close to him/her. Objective diagnostic outcome measures would be a diagnosis on the basis of anatomical 
pathology, and morphological and functional imaging techniques. The aim of the treatment is to improve or slow down the deterioration in cognitive function, improve scores for activities of daily living, and to improve the overall clinical state. A variety of evaluation scales are available for this purpose: Alzheimer's Disease Assessment Scale-Cognitive subscale (ADAS-cog), Global Deterioration Scale (GDS), Clinical Global Impressions (CGI) etc., up to quality-of-life scales.

In neuropsychiatry, the importance of objective outcome measures in the evaluation of treatments is currently marginal; however, how important will they be in the long term, particularly if advances in imaging techniques enable more sensitive and more specific investigation of these diseases?

After analysing these various situations, one can conclude that the importance of subjective outcome measures in drug evaluation is highly variable, and depends on the field of the disease and its particular nature.

Certain general lessons can nevertheless be drawn from the analysis of these various situations:

- Subjective outcome measures are more frequently used in the evaluation of chronic conditions.

- Subjective outcome measures are more often used in curative situations and less often in the evaluation of preventive treatments.

- When an objective outcome measure exists, the subjective outcome measure is not considered in isolation, even if the former appears to be clinically less relevant than the subjective outcome measure. This raises the problem of organising the outcome measures into a hierarchy according to the therapeutic objective, as well as that of the correlation between the outcome measures.

- The use of a subjective outcome measure raises the problem of the specificity of the measure for this disease. The objective outcome measure is 'disease-specific', while the subjective outcome measure takes the 'sick patient' into account.

- The use of subjective outcome measures should be incorporated into guidelines for development. It should be possible to modify the required outcome measures depending on the progress of the therapy.

\section{The Available Scales, Their Validation and Their Metrological Properties}

Although it is impossible to draw up an exhaustive list of available instruments, an inventory of the various types of instrument is interesting because it highlights their great diversity. Thus, we find instruments comprising a single item, e.g. VAS, notably used in the measurement of pain, ${ }^{[12]}$ or clinical global impression scales, ${ }^{[13]}$ commonly used in trials on psychotropic drugs. On the other hand, certain instruments include several hundred items; here, we will mention just one, the MMPI, ${ }^{[14]}$ an instrument for measuring personality.

These instruments can evaluate a targeted symptom, such as dyspnoea $^{[15]}$ or, on the other hand, a multi-factorial concept such as quality of life ${ }^{[16]}$ or satisfaction. ${ }^{[17]}$ Certain instruments give a single score for a single concept measured, e.g. quality of life, ${ }^{[18]}$ and others give a profile score. ${ }^{[19]}$ Most of these measures are self-assessments (the patient answers questions put to him or her, usually by means of a questionnaire), whereas others are assessments made by an observer (in this case, a clinician makes an assessment of the patient). Finally, we should mention that all these instruments can exist in electronic form rather than in the conventional form of a hard-copy questionnaire.

This great diversity in the possible forms the instruments can take implies a certain level of diversity in the methodology that is used to validate them. However, it is possible to present the broad outlines of this validation process. The validation of a subjective measuring instrument relies on two points: setting out the practical methods of its construction and performing an experimental study to evaluate its performance.

In the construction phase, the specifications of the instrument must be stated (the concept to be measured should be defined, as should the target population and the context in which it will be used, etc.). A guide presenting the instrument, in order to explain how to use it, is indispensable. The way data is collected on the series of conceivable items must be made clear and should include details (notably the use of qualitative interviews with patients).

The more experimental phase that aims to complete the validation of the instrument is based on the following assumption: that two questions lie behind the problem of validating a subjective measuring instrument. How is the concept measured by the instrument to be visualised? In other words, what does the instrument measure? What are the metrological properties of the numerical result derived from the measure, i.e. how good is the measure? In order to answer the first question, in addition to a careful examination of the content of the various items that constitute the instrument (content validity), one should look for correlation between the measure being validated and a variety of variables: the concepts of convergent, divergent and predictive validity, etc. are central here. To answer the second question, analyses from the field of psychometrics are required: the key ideas here are internal consistency, interobserver agreement and sensitivity to change.

Such a strategy can be summarised in figure $1 .{ }^{[2]}$ 


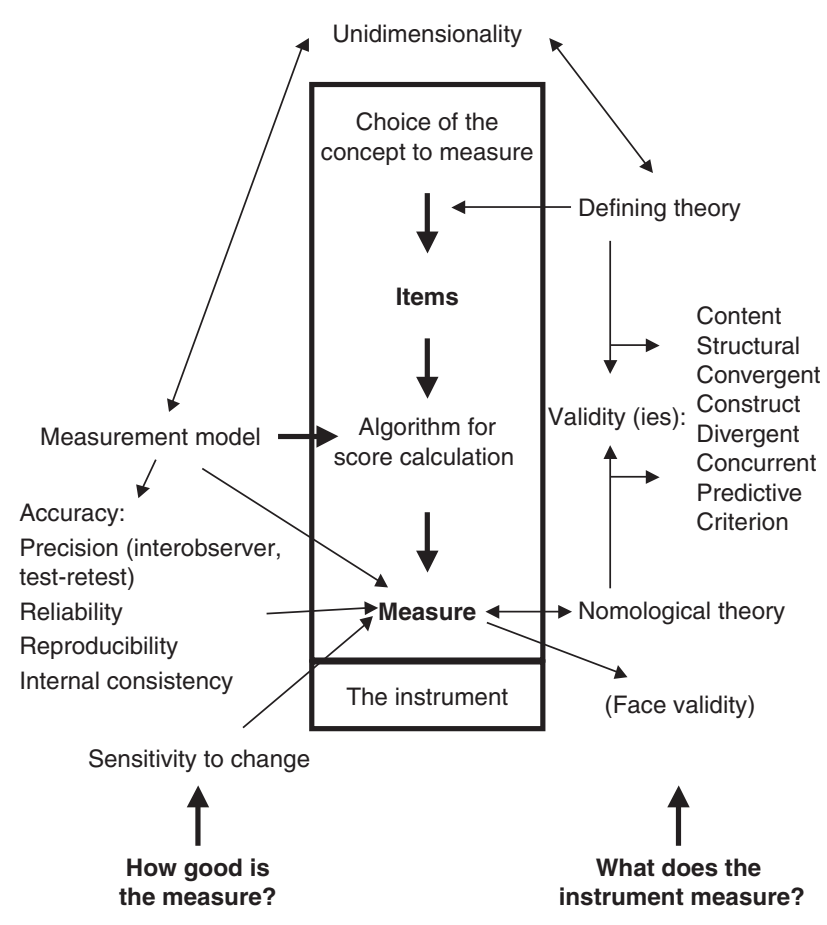

Fig. 1. Validation strategy for a subjective measurement instrument.

Figure 1 should be interpreted in the following way. Validating a subjective measurement instrument involves answering two questions: What does the instrument measure? How good is the measure?

The first question is initially answered by analysing the $a$ priori assumptions that determined the choice of items by those who designed the instrument (defining theory, content validity). It is also answered by comparing the measures obtained from the instrument with measures derived from other instruments, some of which are conceptually close (concurrent validity) or more distant (divergent validity). Studying the dimensional structure of the instrument (factorial analysis, structural validity) is also an important stage.

The answer to the second question (How good is the measure?) is more technical and relies on many statistical methods from the field of psychometrics. The main key words here are internal consistency (Cronbach's coefficient alpha), interobserver agreement or test-retest reliability (intraclass correlation coefficient) and sensitivity to change (effect size).

To summarise, at the end of the experimental phase of validation, one should be able to specify the following: (i) the distribution of the responses to the items; (ii) the dimensional structure of the instrument; (iii) the validity of the measurement model used; (iv) the precision (reliability) of the measure obtained; (v) its sensitivity to change (the statistical tools being unfortunately inadequate in this field); and (vi) the study of the relationship between the measure(s) in question and several other measures, in order to provide support for the precise meaning of the concept being studied (concurrent and divergent validity, etc.).

The number of subjects that should be included in such a study depends essentially on the precision of the main validity parameters that needs to be achieved (Cronbach's coefficient alpha, coefficients of the principal component analysis, correlation coefficients, etc.). In practice, the sample sizes will usually be between 100 and 500 subjects. ${ }^{[2]}$

\section{The Value of Subjective Outcome Measures in Marketing Authorisation Dossiers and in Drug Reassessment After Release onto the Market}

The use of subjective outcome measures in drug evaluation raises certain specific questions. We have attempted to provide an answer to each of these questions.

- What is the importance of subjective outcome measures, as a main outcome measure or as a secondary outcome measure? What is the additional value of subjective outcome measures?

Evaluating efficacy using an objective outcome measure tends to answer a question that is specifically concerned with the disease, whereas evaluating efficacy with a subjective outcome measure tends to answer a question that concerns the patient.

In cases where both types of outcome measures coexist, the objective outcome measure should not necessarily be considered as the main outcome measure, and the importance of the subjective outcome measure must be discussed for each different case, and depends on the aim of the study and the disease in question.

Depending on the disease being studied, the presence or absence of a correlation between the objective outcome measure and the subjective outcome measure should be discussed, in such a way as to clearly show that the subjective outcome measure adds crucial information to the evaluation.

- If scales that measure subjective outcome measures are used in the evaluation of drug efficacy, what sort of validation should be provided?

There are two situations in this case:

1. Either a standard instrument that has previously been validated is used, such as the Hamilton Depression Rating Scale, in which instance it is sufficient to cite references.

2. Or if the instrument is not standard, validation information such as that previously described must be included in the dossier, from the design of the instrument up to its validation. Most problems are encountered at the time of evaluation in such situations, and it is important to be critical of the instrument: Generic instrument versus specific instrument? Was the instrument constructed 
by considering what is known about the drug? What were the validation conditions, etc.?

3. What are the methodological and statistical problems associated with measuring subjective outcome measures?

The same methodological standards should be applied to both subjective and objective outcome measures: Trials should have the same qualities of design, they should be conducted in the same way, and the same rigour should be applied. Double blinding is particularly desirable.

Similarly, identical statistical standards should be applied, whether using objective or subjective outcome measures. Nevertheless, with subjective outcome measures, there are some more specific problems that should be considered, such as how to deal with missing data (often more extensive), as well as that of the multidimensional nature of the evaluation. In any case, these issues should be considered beforehand, and all the details of the analysis and the interpretation of the data should, in principle, be supplied and justified in the analysis plan.

- How to provide a solution to the problem of the clinical significance of the results: The size of the effect?

No completely satisfactory answer can be given to this difficult question from a methodological point of view. In clinical interpretation, statistical significance is not enough. The problem of clinical relevance depends on the disease, on whether the measure is used in patient follow-up procedures, and on the threshold value beyond which there is a medical consensus that improvement or deterioration are clinically significant.

However, several methods (none of which are perfect) can be used and provide observers with information to assist them in their decision. The 'effect size' can be mentioned here, as can converting the results into 'responder rates'.

- What problems are encountered when extrapolating the results?

It can be difficult to extrapolate from one population to another when using subjective measures that rely on questionnaires. Problems related to translation must be dealt with when validating the measurement instrument. Furthermore, cultural differences can alter the sensitivity and significance of a measurement instrument. It may be necessary to conduct a study in the target population.

- What is the specificity of subjective measurement instruments?

The specificity of the measure is strongly associated with the instrument in question.

There is thus a 'spectrum' from the measurement of an isolated symptom (e.g. dyspnoea) through polysymptomatic measures and evaluation of functional capacity, followed by evaluation of handicap, and up to a measure of quality of life, which

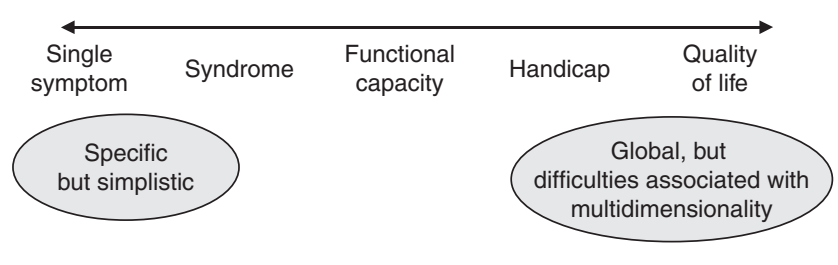

Fig. 2. For subjective measurement instruments, there is a 'spectrum' from the measurement of an isolated symptom up to a measure of quality of life.

involves assessing a wide range of elements perceived by the patient (figure 2). ${ }^{[20]}$

A unidimensional subjective measure is not very different in reality from an objective measure. It is specific but does not assess the patient in a global way. An example is the measurement of one symptom - it is very specific, easy to interpret, but perhaps simplistic.

On the other hand, panels of multidimensional measures take many patient-related elements into account, an extreme example being the quality-of-life scales. When they are properly applied, they provide a global assessment of the patient. However, great care must be taken when interpreting the results, and they can cause problems in analyses because they are multidimensional. ${ }^{[16]}$

In drug evaluation, the aim is to choose precisely the type of instrument best suited to the question that the study should answer, and to have a convincing combination of different assessments.

The ERIQA group (European Regulatory Issues on Quality of Life Assessment), which includes both the pharmaceutical industry and academics, has set itself the task of establishing the principles and practices for integrating quality-of-life measures into the drug registration process. The group aims to ensure the appropriate use of measures in studies, so that clinical value is determined with a view to increasing the acceptance of qualityof-life data by the registration authorities. ${ }^{[21]}$

In conclusion, the following recommendations can be made:

- Subjective outcome measures that are generally used in addition to objective outcome measures can claim the status of the main outcome measure. They are useful and provide indispensable and relevant information for evaluating drug efficacy.

- The instrument used must be validated using an appropriate methodology. This validity must be established in the various countries where the study will be conducted.

- When the main outcome measure is a subjective outcome measure, the study methodology and the statistical analysis plan obey the same rules as when using an objective outcome measure. 
- The issue of the clinical significance of the results must be dealt with and discussed in the dossier in such a way as to give those assessing it the information that was used in the interpretation. The use of unidimensional tools makes interpretation easier (at least in a first approximation); otherwise, it is advisable to specify beforehand the dimension or dimensions of the scale on which the main analysis is based.

- These different types of measure should be considered because they provide a different and complementary perspective on the disease and the patient.

These considerations should be taken into account at the time of the first evaluation of the drug (MA) and at its subsequent reassessment.

\section{Participants}

B. Arnould (MAPI, Lyon), B. Avouac (Hôpital Henri Mondor, Créteil), O. Chassany (Hôpital Lariboisière, Paris), B. Hamelin (Astra-Zeneca, Rueil Malmaison), G. Lapeyre (Pharmacia and Upjohn, Saint Quentin en Yvelines), P. Lendresse (Aventis, Paris), A. Leplège (INSERM U292, Le Kremlin Bicètre), M. Lièvre (UFRRTH, Lyon), H. Mathiex-Fortunet (Beaufour Ipsen Pharma, Paris), G. Paintaud (Hôpital Bretonneau, Tours), M. Pigeon (CNAMTS/DDRI, Paris), A. Puech (Sanofi Synthelabo, Chilly Mazarin), R. Samoyeau (BYK, Le Mée sur Seine), A. Spriet (Alain Spriet Conseil, Paris), G. Steinberg (Produits Roche, Neuilly sur Seine), C. Vilain (GSK, Marly-leRoi).

\section{References}

1. Bouvenot G, Vray M. Essais cliniques : théorie, pratique et critique. Paris: Médecine-sciences Flammarion, 1999

2. Falissard B. Mesurer la subjectivité en santé : perspective méthodologique et statistique. Paris : Masson, 2001

3. Clemens J, Brenner R, Rao M, et al. Evaluating new vaccines for developing countries: efficacy or effectiveness? JAMA 1996; 275: 390-7

4. Dougados M, Lequesne M, Maheu E, et al. L'arthrose en question. Paris : Editions Laboratoires Pharmascience, 1996

5. Felson DT, Anderson JJ, Boers M, et al. American College of Rheumatology: preliminary definition of improvement in rheumatoid arthritis. Arthritis Rheum 1995; 38: 727-35

6. European Agency for the Evaluation of Medicinal Products (EMEA). Points to consider on clinical investigation of medicinal products in the treatment of pa- tients with chronic obstructive pulmonary disease (COPD), CPMP/EWP/562/98 [online]. Available from URL: http://www.emea.eu.int/pdfs/human/ewp/ 056298en.pdf [Accessed 2003 Jul 16]

7. Quantin X, Jacot W, Choma D, et al. Méthodologie des essais cliniques. Rev Pneumol Clin 2001; 57: S13-6

8. European Agency for the Evaluation of Medicinal Products (EMEA). Note for guidance on evaluation of anticancer medicinal products in man, CPMP/EWP/205/95 [online]. Available from URL: http://www.emea.eu.int/pdfs/human/ewp/ 020595en.pdf [Accessed $2003 \mathrm{Jul}$ 16]

9. Geyer CE, Green JS, Moinpour CM, et al. Expanded phase II trial of paclitaxel in metastatic breast cancer: a Southwest Oncology Group Study. Breast Cancer Res Treat 1998; 51: 169-81

10. Bourin M. Clinical methodology for testing of anxiolytic drugs. Thérapie 2000; 55: $147-53$

11. Collins EJ, Hogan TP, Desai H. Measurement of therapeutic response in schizophrenia: a critical survey. Schizophr Res 1991; 5: 249-53

12. Huskisson EC. Measurement of pain. Lancet 1974; II (7889): 1127-31

13. Guelfi JD. L'évaluation clinique standardisée. Castres : Editions Médicales Pierre Fabre, 1993

14. Perse J. Manuel du MMPI. Paris : Editions du CPA, 1986

15. Eakin EG, Resnikoff PM, Prewitt LM, et al. Validation of a new dyspnea measure: the UCSD Shortness of Breath Questionnaire. Chest 1998; 113: 619-24

16. Lepègle A. Les mesures de la qualité de vie. Paris : Presse Universitaires de France (puf) coll. Que sais-je ? : n 3506,1999

17. Salomon L, Gasquet I, Mesbah M, et al. Construction of a scale measuring inpatients 'opinion on quality care'. Int J Qual Health Care 1999; 11: 507-16

18. Drummond MF, O'Brien BJ, Stoddart GL, et al. Méthodes d'évaluation économique des programmes de santé. Paris : Economica, 1998

19. Landgraf JM, Abetz LN, Ware JE. The Child Health Questionnaire (CHQ): a user's manual. Boston (MA): The Health Institute, New England Medical Center, 1996

20. Patrick DL. Concept of health-related quality of life and of patient reported outcomes. In: Chassany $\mathrm{O}$, Caulin $\mathrm{C}$, editors. Qualité de vie liée à l'état de santé, critère d'évaluation. Paris : Springer Verlag, 2002: 23-4

21. Chassany O, Sagnier P, Marquis P, et al. Patient-reported outcomes: the example of health-related quality of life: a European guidance document for the improved integration of Health-Related Quality of Life assessment in the drug approval process: European Regulatory Issues on Quality of Life Assessment (ERIQA) Group. Drug Inf J 2002; 36: 209-38

Correspondence and offprints: Bruno Falissard, INSERM U 472, Hôpital Paul Brousse, 14 Avenue Paul Vaillant Couturier, 94807 Villejuif, France.

E-mail: falissard@vjf.inserm.fr 